\title{
Twisted bialgebroids versus bialgebroids from a Drinfeld twist
}

\author{
Andrzej Borowiec ${ }^{1, *}$ and Anna Pachol', \\ ${ }^{1}$ Institute of Theoretical Physics, University of Wroclaw, pl. M. Borna 9, 50-204 Wroclaw, Poland. \\ ${ }^{2}$ Queen Mary, University of London, School of Mathematics, Mile End Rd., London E1 4NS, UK.
}

\begin{abstract}
Bialgebroids (resp. Hopf algebroids) are bialgebras (Hopf algebras) over noncommutative rings. Drinfeld twist techniques are particularly useful in the (deformation) quantization of Lie algebras as well as underlying module algebras (=quantum spaces). Smash product construction combines these two into the new algebra which, in fact, does not depend on the twist. However, we can turn it into bialgebroid in the twist dependent way. Alternatively, one can use Drinfeld twist techniques in a category of bialgebroids. We show that both techniques indicated in the title: twisting of a bialgebroid or constructing a bialgebroid from the twisted bialgebra give rise to the same result in the case of normalized cocycle twist. This can be useful for better description of a quantum deformed phase space. We argue that within this bialgebroid framework one can justify the use of deformed coordinates (i.e. spacetime noncommutativity) which are frequently postulated in order to explain quantum gravity effects.
\end{abstract}

\section{INTRODUCTION}

Quantum groups and Hopf algebras over the years have proved their important role in approach to Quantum Gravity. They are considered as one of the tools of the Noncommutative Geometry which introduces more general idea of the geometry and allows for a natural quantization of manifolds (e.g. spacetime). In some recent works [1], 2] the idea of generalization of Hopf algebras into Hopf algebroids [3, 5] in the quantum spacetimes context have been approached. It is rather well known that the unification of spacetime coordinates with a Lie algebra of symmetries cannot be done within the category of Lie algebras. The corresponding construction is called smash (or more generally cross) product which requires introducing the Hopf algebra framework. The algebra of spacetime coordinates is then Hopf module algebra. A special example of this construction is provided by the so-called phase space algebra (as the Heisenberg algebra in Quantum Mechanics). The phase space algebra cannot be equipped with the Hopf algebra structure (at most it can be made into unital-non-counital bialgebra). However such smash product can be generalized into the bialgebroid. This approach leads to more sophisticated algebraic structures as Hopf algebroids [], , [5], [6], , [7], [8] Recently the quantum (deformed) phase spaces with the noncommutative coordinates equipped with the bialgebroid (Hopf algebroid) structures gained some attention in the mathematical physics literature.

Deformed quantum phase spaces have been considered shortly after the noncommutative spacetimes were introduced, especially in the context of the $\kappa$-deformation [9]. A natural extension of the $\kappa$-Poincare quantum group [9, 10] by the $\kappa$-Minkowski commutation relations contains deformation of the Heisenberg subalgebra (phase space). There have been many constructions of such deformed phase space, e.g. within Heisenberg double construction [1] or smash product construction [12, 13]. Deformed quantum phase spaces are constantly studied with a number of interesting papers appearing recently, like e.g. 14].

Focusing on the special case of deformed quantum phase spaces with the noncommutative coordinates satisfying the $\kappa$-deformed Minkowski algebra [10, 15], the Hopf algebroid structure was firstly investigated in [1]. Later on the more detailed study on the covariance of such space under the action of the $\kappa$-deformed Poincare symmetry within the Hopf algebroid framework was proposed with the Heisenberg double construction naturally providing the bialgebroid structure [2]. Still the physical meaning of Hopf algebroids stays not entirely clear in this context and requires more studies in this direction. We believe that within the bialgebroid framework one can better justify the deformed Casimir relations which are frequently used in order to explain some quantum gravity effects coming from the spacetime noncommutativity.

Hopf algebroids are Hopf algebras over unital noncommutative rings. One of the oldest definitions of bialgebroids go back to Sweedler [6] and Takeuchi 7]. Schauenberg [4] also contributed to the topic with his paper on bialgebras over the noncommutative rings. However the concept of Hopf algebroids (bialgebroids with an antipode) was introduced by $\mathrm{Lu}[3]$ in 1996. The concept of twisted bialgebroids was firstly considered by $\mathrm{Xu}$ in 2000 [5]. Lu's definition came as a result of work on grupoids in Poisson geometry whereas the one by $\mathrm{Xu}$ came from quantum universal enveloping

*Electronic address: andrzej.borowiec@ift.uni.wroc.pl

${ }^{\dagger}$ Electronic address: a.pachol@qmul.ac.uk 
algebroids (quantum groupoids) point of view. It was later shown [16] that these two definitions are equivalent. In 2004, G. Böhm and K. Szlachanyi [8] considered pairs of bialgebroids on which the antipode map was defined.

We will be interested in the Drinfeld twist techniques which are particularly useful in the (deformation) quantization of (complex or real) Lie algebras as well as underlying module algebras (=quantum spaces). The smash product construction combines these two into a new algebra which, in fact, remains isomorphic to itself under twisting. However, if this algebra is turned into a bi- (or Hopf) algebroid, the latter is no longer isomorphic under twisting.

Our aim in this note is to show the equivalence between the bialgebroid obtained as a result of the smash product of a twisted triangular bialgebra with the twisted braided commutative module algebra [16] and the one obtained as a result of twisting of the smash product in the category of bialgebroids [5].

\section{PRELIMINARIES AND NOTATIONS}

In this note we shall work in a category of $K$-modules, where $K$ is a base commutative ring with unit $1 \equiv 1_{K}$. Therefore all objects are by default $K$-modules, all maps are $K$-linear maps. The tensor $\otimes$ product if not indicated otherwise is over the ring $K$. Particularly interesting cases are when $K=\mathbb{K}$ is a field (of characteristic 0 ) or $K=\mathbb{K}[[h]]$ is a (topological) ring of formal power series in the (formal) variable $h$. All rings (algebras) are assumed to be unital. All modules and module maps are assumed to respect the unit. Below, for the notational convenience, we shall briefly introduce the main notions involved in our presentation. For deeper study we refer the reader to the literature.

\section{A. Smash Product Construction [17-19]}

Let $\mathcal{H}=\left(H, \Delta, \epsilon, 1_{H}\right)$ be a bialgebra and $\mathcal{A}=\left(A, \star, 1_{A}\right)$ be a left $H$-module algebra with the action $\triangleright: H \otimes A \rightarrow A$ such that: $1_{H} \triangleright a=a, M \triangleright 1_{A}=\epsilon(M) 1_{A}$. Smash product algebra $A \rtimes H$ is an algebra determined on the vector space $A \otimes H$ by the multiplication $(a \otimes L)(b \otimes J)=a\left(L_{(1)} \triangleright b\right) \otimes L_{(2)} J$, where $a, b \in A ; L, J \in H$ and $\Delta(L)=L_{(1)} \otimes L_{(2)}$ in Sweedler shortcut notation. Obviously, the algebra $A \rtimes H$ contains algebras $A \ni a \mapsto a \otimes 1 \in A \rtimes H$ and $H \ni L \mapsto 1 \otimes L \in A \rtimes H$ as subalgebras. Later on we shall denote by $a \rtimes L$ elements from $A \rtimes H$ of the form $a \otimes L$. Therefore the previous formula can be rewritten as

$$
(a \rtimes L)(b \rtimes J)=a\left(L_{(1)} \triangleright b\right) \rtimes L_{(2)} J .
$$

A special case of this construction provides the algebra of canonical commutation relations between commuting coordinates and momenta generators (see e.g. [20] and references therein), which are fundamental from the point of view of Quantum Mechanics (quantum phase space). In the physically motivated examples this algebra is further extended by the presence of symmetry, e.g. Lorentz generators, which together with the position and momentum generators form the so-called extended spacetime-Poincaré algebra (also called the extended phase space, see e.g. [13] and the references therein). Various applications to the description of Quantum Gravity effects rely on a suitable (quantum) deformation of both a coordinate algebra as well as a corresponding symmetry (Hopf) algebra (see e.g. [21]). In such cases besides the traditional position-momentum noncommutativity one postulates following 23, 24] also the noncommutativity between position variables and/or, less frequently, between momentum variables. Such kind of theories can be also considered as noncommutative versions of Quantum Mechanics [25, 26]. Drinfeld twist techniques turn out to be useful tool in their construction. This point will be a subject of the present note.

\section{B. Quasitriangular Hopf Algebras [27, 28] and Drinfeld twist techniques 29]}

Let $(\mathcal{H}, R)$ be a quasi-triangular bialgebra with the universal quantum R-matrix $R=R_{1} \otimes R_{2} \in H \otimes H$ satisfying

$$
R \Delta(X) R^{-1}=\Delta^{o p}(X), \quad(\Delta \otimes i d) R=R_{13} R_{23}, \quad(i d \otimes \Delta) R=R_{13} R_{12}, \quad(\epsilon \otimes i d) R=(i d \otimes \epsilon) R=1
$$

which imply quantum Yang-Baxter equation

$$
R_{12} R_{13} R_{23}=R_{23} R_{13} R_{12}
$$


As is known [30], 31] any (left) module $A$ over $(\mathcal{H}, R)$ becomes automatically a (left-right) Yetter-Drinfeld module with the right coaction $\delta_{R}(a)=\left(R_{2} \triangleright a\right) \otimes R_{1}$ for all $a \in A .^{1}$

The category of all (left-right) Yetter-Drinfeld modules $\mathcal{H} \mathfrak{Y} \mathfrak{D}^{\mathcal{H}}$ is a prebraided (and braided if $\mathcal{H}$ is a Hopf algebra) monoidal category [32]. In particular, a left $H$ - module algebra $\mathcal{A}=\left(A, \star, 1_{A}\right)$ is an algebra in $\mathcal{H} \mathfrak{Y} \mathfrak{D}^{\mathcal{H}}$ if and only if it is a braided commutative, i.e.

$$
a \star b=\left(R_{2} \triangleright b\right) \star\left(R_{1} \triangleright a\right)
$$

Let $F \in H \otimes H$ be a normalized cocycle twist in $(\mathcal{H}, R)$, i.e an invertible element which satisfies the following conditions

$$
\begin{gathered}
F_{12}(\Delta \otimes i d)(F)=F_{23}(i d \otimes \Delta)(F) \Leftrightarrow F_{1^{\prime}}\left(F_{1}\right)_{(1)} \otimes F_{2^{\prime}}\left(F_{1}\right)_{(2)} \otimes F_{2}=F_{1} \otimes F_{1^{\prime}}\left(F_{2}\right)_{(1)} \otimes F_{2^{\prime}}\left(F_{2}\right)_{(2)} \\
(\epsilon \otimes i d)(F)=1_{H} \otimes 1_{H}=(i d \otimes \epsilon(F)
\end{gathered}
$$

Its inverse satisfies the similar conditions (according to our notation $F=F_{1} \otimes F_{2}, \quad F^{-1}=\bar{F}_{1} \otimes \bar{F}_{2}$ ):

$$
\begin{gathered}
\left((\Delta \otimes i d) F^{-1}\right) F_{12}^{-1}=\left((i d \otimes \Delta) F^{-1}\right) F_{23}^{-1} \Leftrightarrow\left(\bar{F}_{1}\right)_{(1)} \bar{F}_{1^{\prime}} \otimes\left(\bar{F}_{1}\right)_{(2)} \bar{F}_{2^{\prime}} \otimes \bar{F}_{2}=\bar{F}_{1} \otimes\left(\bar{F}_{2}\right)_{(1)} \bar{F}_{1^{\prime}} \otimes\left(\bar{F}_{2}\right)_{(2)} \bar{F}_{2^{\prime}} \\
(\epsilon \otimes i d) F^{-1}=1_{H} \otimes 1_{H}=(i d \otimes \epsilon) F^{-1}
\end{gathered}
$$

The twisting element serves the purpose of deformation both the bialgebra structure $\Delta \mapsto \Delta^{F}=F \Delta F^{-1}$ as well the corresponding module algebra structure $\star \mapsto \star_{F}=\star \circ\left(\bar{F}_{1} \triangleright \otimes \bar{F}_{2} \triangleright\right)$. We shall denote these new algebras as $\mathcal{H}^{F}=\left(H, \Delta^{F}, \epsilon\right)$ and $\mathcal{A}_{F}=\left(A, \star_{F}\right)$. Moreover, $\left(\mathcal{H}^{F}, R^{F} \equiv F_{21} R F^{-1}\right)$ is quasi-triangular and the module algebra $\left(A, \star_{F}\right) \in \mathcal{H}^{F} \mathfrak{Y} \mathfrak{D}^{\mathcal{H}^{F}}$ if and only if $(A, \star) \in \mathcal{H} \mathfrak{Y} \mathfrak{D}^{\mathcal{H}}$. Drinfeld twisting techniques are very useful in mathematical physics, noncommutative geometry (see e.g [33]) when looking for new quantum spaces and their quantum symmetries (e.g. [34]).

\section{Bialgebroids $[3,5,8]$}

The bialgebroid $\mathcal{M}=(M, A, s, t, \Delta, \epsilon)$ consists of a total algebra $M$ and a base algebra $A$ and the following data:

B1) Two mappings: an algebra homomorphism $s: A \rightarrow M$ called a source map and an algebra anti-homomorphism $t: A \rightarrow M$ called a target map such that: $s(a) t(b)=t(b) s(a)$ is satisfied for all $a, b \in A$.

We consider a left bialgebroid $\mathcal{M}$ as an $A$-bimodule (with the bimodule structure which prefers the left side) as follows: $a . m . b=s(a) t(b) m$ for all $a, b \in A, m \in M$.

B2) Additionally, it is equipped with coproduct and counit maps. Coproduct and counit make $M$ an $A$-coring [16] (with axioms like that of a coalgebra such that all mappings are $A$-bimodule homomorphisms and all tensors are over A).

More exactly, the bialgebroid coproduct map $\Delta: M \rightarrow M \otimes_{A} M$ is an $A$-bimodule map, where $M \otimes_{A} M$ is constructed in such a way that $(t(a) m) \otimes_{A} n=m \otimes_{A}(s(a) n)$; simplifying the notation one can write $(m . a) \otimes_{A} n=m \otimes_{A}(a . n)$. This is due to the fact that as an Abelian (additive) group $M \otimes_{A} M$ is a quotient group of $M \otimes M$ by a subgroup generated by the elements $\{(t(a) \otimes 1-1 \otimes s(a)) m \otimes n: a \in A, m, n \in M\}$. This subgroup is, in fact, a left ideal in the algebra $M \otimes M$. However, $M \otimes_{A} M$ (unlike $M \otimes M$ ) is not an algebra in general. To fix this problem one introduces the so-called Takeuchi product $M \times_{A} M$ [7]. ${ }^{2}$ It is defined as a subgroup of invariant elements $M \times_{A} M=\left\{m \otimes_{A} n \in M \otimes_{A} M:(m t(a)) \otimes_{A} n=m \otimes_{A} n(s(a)) ; \forall a \in A\right\}$ which has natural (componentwise) multiplication $\left(\left(m \otimes_{A} n\right)\left(p \otimes_{A} q\right)=m p \otimes_{A} n q\right)$. Both $M \otimes_{A} M$ and $M \times_{A} M$ inherit $A$-bimodule structure determined by the action $m \otimes_{A} n \mapsto(s(a) m) \otimes_{A}(t(b) n)$, or $a .\left(m \otimes_{A} n\right) . b=(a . m) \otimes_{A}(n . b)$. Now we can request additionally that the image of the coproduct map is in $M \times_{A} M$, i.e. that one deals, in fact, with the algebra map: $\Delta(m n)=\Delta(m) \Delta(n) \equiv m_{(1)} n_{(1)} \otimes_{A} m_{(2)} n_{(2)}$

1 Throughout the paper we shall be using a shorthand notation of Sweedler type. The coproduct is denoted as $\Delta(L)=L_{(1)} \otimes L_{(2)}$. For elements $R \in H \otimes H$ we write $R=R_{1} \otimes R_{2}$, If $R$ is invertible we write $R^{-1}=\bar{R}_{1} \otimes \bar{R}_{2}: \bar{R}_{1} R_{1^{\prime}} \otimes \bar{R}_{2} R_{2^{\prime}}=\bar{R}_{1^{\prime}} R_{1} \otimes \bar{R}_{2^{\prime}} R_{2}=1_{H} \otimes 1_{H}$.

2 We follow notational convention introduced in the previous section also for elements of $M \otimes_{A} M$. 
The counit map $\epsilon: M \rightarrow A$ has to satisfy:

$$
\epsilon\left(1_{M}\right)=1_{A}, \quad \epsilon(m n)=\epsilon(m s(\epsilon(n)))=\epsilon(m t(\epsilon(n))), \quad s\left(\epsilon\left(m_{(1)}\right)\right) m_{(2)}=t\left(\epsilon\left(m_{(2)}\right)\right) m_{(1)}=m
$$

The axioms [3] are similar to those of a bialgebra but are complicated by the possibility that $A$ is a noncommutative algebra, instead of a commutative $\operatorname{ring} K$, or its images under $s$ and $t$ are not in the center of $M$.

In a case of Hopf algebroids, one additionally assumes that an antipode $\tau: M \rightarrow M$ is to be an algebra antiautomorphism satisfying conditions of exchanging the source and target maps and satisfying two axioms similar to the Hopf algebra antipode axioms. The different versions of introducing the antipode map are possible (see e.g. the second reference in 8]).

We recall that a morphism between two bialgebroids: $(M, A, s, t, \Delta, \epsilon)$ and $\left(M^{\prime}, A, s^{\prime}, t^{\prime}, \Delta^{\prime}, \epsilon^{\prime}\right)$ over the same algebra $A$ consists of algebra map $\phi: M \rightarrow M^{\prime}$ such that $\phi \circ s=s^{\prime}, \phi \circ t=t^{\prime}, \epsilon=\epsilon^{\prime} \circ \phi$ and the following diagram commutes

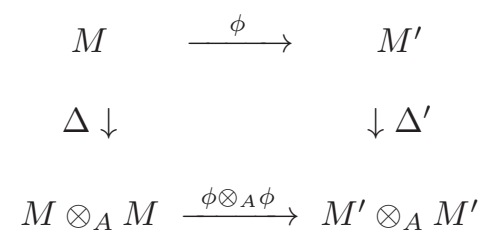

i.e. $\Delta^{\prime} \circ \phi=\left(\phi \otimes_{A} \phi\right) \circ \Delta$. For the case of Hopf algebroids with an antipode $\tau$ one should also assume that $\tau^{\prime} \circ \phi=\phi \circ \tau$.

\section{Smash product algebras as bialgebroids [16]}

In [16] it was shown how a Hopf algebroid structure can be associated to a smash product of a Hopf algebra with a braided commutative algebra in the Yetter-Drinfeld $\mathfrak{Y} \mathfrak{D}$ category. The theorem [Theorem 4.1 in [16]] adapted to our needs reads as follows:

Theorem II.1 Let $\mathcal{H}=(H, \Delta, \epsilon)$ be a bialgebra, $\mathcal{A}=(A, \star)$ is a left $\mathcal{H}$-module algebra and $(\mathcal{A}, \rho)$ a right $\mathcal{H}$-comodule .

If $(A, \star, \rho)^{3}$ is a braided commutative algebra in $\mathcal{H} \mathfrak{Y} \mathfrak{D}^{\mathcal{H}}$ then $(\mathcal{A} \rtimes \mathcal{H}, s, t, \tilde{\Delta}, \tilde{\epsilon})$ is an $\mathcal{A}$-bialgebroid with the source, target, coproduct and the counit given by the following maps:

$$
\begin{aligned}
s(a) & =a \rtimes 1_{H}, \quad t(a) \equiv \rho(a)=a_{<0>} \rtimes a_{<1>} \\
\tilde{\Delta}(a \rtimes L) & =\left(a \rtimes L_{(1)}\right) \otimes_{\mathcal{A}}\left(1_{A} \rtimes L_{(2)}\right) \\
\tilde{\epsilon}(a \rtimes L) & =\epsilon(L) a
\end{aligned}
$$

for all $a \in A$ and $L \in H$.

Thus $(a \rtimes L) \triangleright b=a \star(L \triangleright b)$. In particular, $\left(a \rtimes 1_{H}\right) \triangleright b=a \star b$ acts by multiplication from the left, while $\left(1_{A} \rtimes L\right) \triangleright b=L \triangleright b$ preserves the initial action.

\section{E. Twisted bialgebroids [5]}

The category of bialgebroids (Hopf algebroids) was introduced by $\mathrm{P}$. Xu in [5] ${ }^{4}$. Let us recall the relevant results from [5] on twist deformation of bialgebroids. Before proceeding further one should remember that bialgebroid definition provides a canonical action $>: M \otimes A \rightarrow A:{ }^{5}$

$$
m \triangleright a=\epsilon(m s(a))=\epsilon(m t(a))
$$

\footnotetext{
$3 \rho$ is an algebra map called coaction: $\rho: A \rightarrow A \otimes H^{o p}, \rho(a)=a<0>\otimes a<1>$ in Sweedler notation.

4 It should be noted that Xu's paper concerns, in fact, bialgebroids (his definition does not include the antipode map).

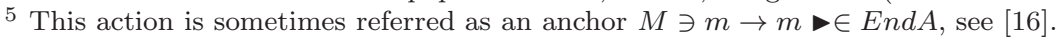


induced by the counit $\epsilon$ (cf. (9)). We should point out that the multiplication in $M$ does not change like in the case of Drinfeld theory, where twist deformation modifies coalgebraic sector only. Nevertheless the one in $A$ changes $\left(A \mapsto A_{F}\right)$ :

$$
\cdot \mapsto \cdot F=\cdot \circ\left(\bar{F}_{1}>\otimes \bar{F}_{2} \triangleright\right)
$$

We are now in position to present the simplified version of [Theorem. 4.14 in [5]], skipping some details which are not relevant for our considerations (following Drinfeld convention our twist is inverse with respect to the one considered by $\mathrm{Xu}$ in [5].):

Theorem II.2 Assume that $(M, A, s, t, \Delta, \epsilon)$ is bialgebroid over the algebra $A$ and $F=F_{1} \otimes_{A} F_{2} \in M \otimes_{A} M$ is a "twistor" (Hopf algebroid twist $\left.{ }^{6}\right)$. Then $\left(M, A_{F}, s_{F}, t_{F}, \Delta_{F}, \epsilon\right)$ is a bialgebroid over the algebra $A_{F}$, where

$$
s_{F}(a)=s\left(\bar{F}_{1}>a\right) \bar{F}_{2} \quad ; \quad t_{F}(a)=t\left(\bar{F}_{2}>a\right) \bar{F}_{1} \quad \forall a \in A .
$$

and new twisted coproduct $\Delta_{F}: M \rightarrow M \otimes_{A_{F}} M:$

$$
\Delta_{F}(m)=F^{\#}\left(\Delta(m) F^{-1}\right), \quad \forall m \in M
$$

The map $F^{\#}: M \otimes_{A} M \rightarrow M \otimes_{A_{F}} M$ is defined by (cf. Corollary 4.4 in [5]):

$$
F^{\#}\left(m \otimes_{A} n\right)=\left(F_{1} m\right) \otimes_{A_{F}}\left(F_{2} n\right) .
$$

\section{MAIN RESULT}

In this section we are going to revisit bialgebroids in a context of twist deformation of smash product algebras . It appears according to construction of Brzezinski-Militaru from [16] that the smash product algebras, under suitable assumptions, can be equipped with the bialgebroid structures. Moreover, we are going to show that the bialgebroid obtained by bialgebroid twisting [5] of the smash product algebra and bialgebroid obtained from the smash product algebra of twisted bialgebra with its twisted module algebra are equivalent (isomorphic).

Let $\mathcal{H} \equiv(H, \Delta, \epsilon)$ be a bialgebra and $\mathcal{A} \equiv(A, \star)$ be a (left) module algebra over $\mathcal{H}$. We denote the corresponding (left) action as $\triangleright: H \otimes A \rightarrow A: L \triangleright(a \star b)=\left(L_{(1)} \triangleright a\right) \star\left(L_{(2)} \triangleright b\right)$. Assume that $F=F_{1} \otimes F_{2} \in H \otimes H$ is a normalized cocycle twist for $\mathcal{H}$. It allows us to construct new bialgebra $\mathcal{H}^{F} \equiv\left(H, \Delta^{F}, \epsilon\right)$ and new module algebra $\mathcal{A}_{F} \equiv\left(A, \star_{F}\right)$ with the same action, where $\Delta^{F}=F \Delta F^{-1}$ and $\star_{F}=\star\left(F^{-1} \circ(\triangleright \otimes \triangleright)\right)$ with $F^{-1}=\bar{F}_{1} \otimes \bar{F}_{2}$. Actually, as far as smash product is concerned, it turns out that:

Proposition III.1 For any Drinfel'd twist $F$ two smash product algebras $\mathcal{A} \rtimes \mathcal{H}$ and $\mathcal{A}_{F} \rtimes \mathcal{H}^{F}$ are isomorphic, even though the algebras $\mathcal{A}$ and $\mathcal{A}^{F}$ are not isomorphic and $\mathcal{H}$ and $\mathcal{H}^{F}$ are not isomorphic as bialgebras (see e.g. [13]).

Although this fact seems to be known, e.g. 35], we provide the proof for completeness.

Proof:

Firstly, we recall that both algebras are determined on the same $K$-module $A \otimes H$ but differ by the multiplications (cf. (11) ):

$$
(a \rtimes L) \star(b \rtimes J)=a \star\left(L_{(1)} \triangleright b\right) \rtimes L_{(2)} J, \quad(a \rtimes L) \star_{F}(b \rtimes J)=a \star_{F}\left(L_{\left(1^{F}\right)} \triangleright b\right) \rtimes L_{\left(2^{F}\right)} J
$$

where $\Delta^{F}(L)=F \Delta(L) F^{-1}=L_{\left(1^{F}\right)} \otimes L_{\left(2^{F}\right)}=F_{1} L_{(1)} \bar{F}_{1^{\prime}} \otimes F_{2} L_{(2)} \bar{F}_{2^{\prime}}$ is the twisted coproduct of the bialgebra $\mathcal{H}^{F}$. Both algebras are generated by simpler elements: $a \rtimes 1_{H}, a \in A$ and $1_{A} \rtimes L, L \in H$, i.e. $a \rtimes L=\left(a \rtimes 1_{H}\right) \star\left(1_{A} \rtimes L\right)=$ $\left(a \rtimes 1_{H}\right) \star_{F}\left(1_{A} \rtimes L\right)$. Of course, the unit $1_{A} \rtimes 1_{H}$ is the same for both multiplications.

The isomorphism $\varphi: \mathcal{A}_{F} \rtimes \mathcal{H}^{F} \rightarrow \mathcal{A} \rtimes \mathcal{H}$ can be defined by the formula

$$
\varphi(a \rtimes L)=\left(\bar{F}_{1} \triangleright a\right) \rtimes \bar{F}_{2} L
$$

such that:

$$
\varphi\left((a \rtimes L) \star_{F}(b \rtimes J)\right)=\varphi(a \rtimes L) \star \varphi(b \rtimes J)
$$

\footnotetext{
${ }^{6}$ It satisfies the same Drinfeld conditions (5) - (8) with $\otimes$ replaced by $\otimes_{A}$.
} 
for all $a, b \in A$ and $L, J \in H$. One notices that due to the normalization condition $\varphi\left(1_{A} \rtimes L\right)=1_{A} \rtimes L$. The inverse $\operatorname{map} \varphi^{-1}: \mathcal{A} \rtimes \mathcal{H} \rightarrow \mathcal{A}_{F} \rtimes \mathcal{H}^{F}$ is, of course, given by $\varphi^{-1}(a \rtimes L)=\left(F_{1} \triangleright a\right) \rtimes F_{2} L$.

We begin by checking the equality (20) for some special cases. Firstly we take

i) $\varphi\left(\left(a \rtimes 1_{H}\right) \star_{F}(b \rtimes J)\right)=\varphi(a \rtimes 1) \star \varphi(b \rtimes J)$.

In $\mathcal{A}_{F} \rtimes \mathcal{H}^{F}: \quad(a \rtimes 1) \star_{F}(b \rtimes J)=\left(a \star_{F} b\right) \rtimes J$ and in $\mathcal{A} \rtimes \mathcal{H}: \quad\left(a \rtimes 1_{H}\right) \star(b \rtimes J)=(a \star b) \rtimes J$.

On one hand, from the above and from (20) we have the following series of equalities:

$$
\begin{aligned}
\varphi\left(\left(a \rtimes 1_{H}\right) \star_{F}(b \rtimes J)\right) & =\varphi\left(\left(a \star_{F} b\right) \rtimes J\right)= \\
\left(\bar{F}_{1} \triangleright\left(a \star_{F} b\right)\right) \rtimes \bar{F}_{2} J & =\bar{F}_{1} \triangleright\left[\left(\bar{F}_{1^{\prime}} \triangleright a\right) \star\left(\bar{F}_{2^{\prime}} \triangleright b\right)\right] \otimes \bar{F}_{2} J= \\
& =\left[\left(\bar{F}_{1}\right)_{(1)} \bar{F}_{1^{\prime}} \triangleright a\right] \star\left[\left(\bar{F}_{1}\right)_{(2)} \bar{F}_{2^{\prime}} \triangleright b\right] \rtimes \bar{F}_{2} J= \\
& =\left(\bar{F}_{1} \triangleright a\right) \star\left(\left(\bar{F}_{2}\right)_{(1)} \bar{F}_{1^{\prime}} \triangleright b\right) \rtimes\left(\bar{F}_{2}\right)_{(2)} \bar{F}_{2^{\prime}} J
\end{aligned}
$$

where we used the cocycle identity for the inverse twist (7).

On the other hand we have from (20) and the above:

$$
\begin{aligned}
\varphi\left(a \rtimes 1_{H}\right) \star \varphi(b \rtimes J) & =\left[\left(\bar{F}_{1} \triangleright a\right) \rtimes \bar{F}_{2}\right] \star\left[\left(\bar{F}_{1^{\prime}} \triangleright b\right) \rtimes \bar{F}_{2^{\prime}} J\right]= \\
& =\left(\bar{F}_{1} \triangleright a\right) \star\left(\left(\bar{F}_{2}\right)_{(1)} \bar{F}_{1^{\prime}} \triangleright b\right) \rtimes\left(\bar{F}_{2}\right)_{(2)} \bar{F}_{2^{\prime}} J= \\
& =\varphi\left(\left(a \rtimes 1_{H} \star_{F}(b \rtimes J)\right)\right.
\end{aligned}
$$

As a next step we consider

ii) $\varphi\left(\left(1_{A} \rtimes L\right) \star_{F}(b \rtimes J)\right)=\varphi\left(1_{A} \rtimes L\right) \star \varphi(b \rtimes J)$

In $\mathcal{A}_{F} \rtimes \mathcal{H}^{F}$ one calculates:

$$
\left(1_{A} \rtimes L\right) \star_{F}(b \rtimes J)=\left(L_{\left(1^{F}\right)} \triangleright b\right) \rtimes L_{\left(2^{F}\right)} J=\left(F_{1} L_{(1)} \bar{F}_{1^{\prime}} \triangleright b\right) \rtimes F_{2} L_{(2)} \bar{F}_{2^{\prime}} J=\varphi^{-1}\left(\left(L_{(1)} \bar{F}_{1^{\prime}} \triangleright b\right) \rtimes L_{(2)} \bar{F}_{2^{\prime}} J\right)
$$

where the first equality is due to the normalization condition $\epsilon\left(\bar{F}_{1}\right) \bar{F}_{2}=1_{H}=\bar{F}_{1} \epsilon\left(\bar{F}_{2}\right)$. Therefore one has the following equalities:

$$
\begin{aligned}
\varphi\left((1 \rtimes L) \star_{F}(b \rtimes J)\right) & =\left(L_{(1)} \bar{F}_{1^{\prime}} \triangleright b\right) \rtimes L_{(2)} \bar{F}_{2^{\prime}} J=\left(1_{A} \rtimes L\right) \star\left(\left(\bar{F}_{1^{\prime}} \triangleright b\right) \rtimes \bar{F}_{2^{\prime}} J\right) \\
& =\varphi\left(1_{A} \rtimes L\right) \star \varphi(b \rtimes J)
\end{aligned}
$$

Verification on the remaining pair of generators: $\left(a \rtimes 1_{H}\right) \star_{F}\left(1_{A} \rtimes J\right)=a \rtimes J$ and $\left(1_{A} \rtimes L\right) \star_{F}\left(1_{A} \rtimes J\right)=1_{A} \rtimes L J$ is rather straightforward.

To finish the proof one checks (20) using above partial results:

$$
\begin{aligned}
\varphi\left((a \rtimes L) \star_{F}(b \rtimes J)\right) & =\varphi\left(\left(a \rtimes 1_{H}\right) \star_{F}\left(\left(\tilde{L}_{(1)} \triangleright b\right) \rtimes \tilde{L}_{(2)} J\right)\right)=\varphi\left(a \rtimes 1_{H}\right) \star \varphi\left(\left(\tilde{L}_{(1)} \triangleright b\right) \rtimes \tilde{L}_{(2)} J\right) \\
& =\varphi\left(a \rtimes 1_{H}\right) \star \varphi\left(\left(1_{A} \rtimes L\right) \star_{F}(b \rtimes J)\right)=\varphi\left(a \rtimes 1_{H}\right) \star \varphi\left(1_{A} \rtimes L\right) \star \varphi(b \rtimes J) \\
& =\varphi(a \rtimes L) \star \varphi(b \rtimes J)
\end{aligned}
$$

The proof is done.

From now on we assume that $(\mathcal{H}, R)$ is quasi-triangular bialgebra and the algebra $\mathcal{A}$ is braided commutative in the category $\mathcal{H} \mathfrak{Y} \mathfrak{D}^{\mathcal{H}}$, i.e. $a \star b=\left(R_{2} \triangleright b\right) \star\left(R_{1} \triangleright a\right)$. Then according to Brzezinski-Militaru construction (Theorem I.1, [16]) $\mathcal{A} \rtimes \mathcal{H}$ is a bialgebroid over the algebra $\mathcal{A}$ if we define (shifting $\Delta: H \rightarrow H \otimes H$ to $\tilde{\Delta}: \mathcal{A} \rtimes \mathcal{H} \rightarrow(\mathcal{A} \rtimes \mathcal{H}) \otimes \mathcal{A}(\mathcal{A} \rtimes \mathcal{H})$ )

$$
\tilde{\Delta}(a \rtimes L)=\left(a \rtimes L_{(1)}\right) \otimes_{\mathcal{A}}\left(1_{A} \rtimes L_{(2)}\right), \quad s(a)=a \rtimes 1_{H}, \quad t(a)=\left(R_{2} \triangleright a\right) \rtimes R_{1}, \quad \tilde{\epsilon}(a \rtimes L)=\epsilon(L) a
$$

The bialgebroid counit map $\tilde{\epsilon}$ will not change through the rest of this note. Following the same idea we can shift the quantum R-matrix from the bialgebra $\mathcal{H}$ to the bialgebroid $\mathcal{A} \rtimes \mathcal{H}$

$$
R \rightarrow \tilde{R}=\left(1_{A} \rtimes R_{1}\right) \otimes_{\mathcal{A}}\left(1_{A} \rtimes R_{2}\right) \in(\mathcal{A} \rtimes \mathcal{H}) \otimes_{\mathcal{A}}(\mathcal{A} \rtimes \mathcal{H}) .
$$


One can easily check by direct calculations that properties of the cocycle type will be preserved (cf. (2)):

$$
\left(\tilde{\Delta} \otimes_{\mathcal{A}} i d\right) \tilde{R}=\tilde{R}_{13} \tilde{R}_{23}, \quad\left(i d \otimes_{\mathcal{A}} \tilde{\Delta}\right) \tilde{R}=\tilde{R}_{13} \tilde{R}_{12}, \quad\left(\tilde{\epsilon} \otimes_{\mathcal{A}} i d\right) \tilde{R}=\left(i d \otimes_{\mathcal{A}} \tilde{\epsilon}\right) \tilde{R}=\left(1_{A} \rtimes 1_{H}\right) \otimes_{\mathcal{A}}\left(1_{A} \rtimes 1_{H}\right)
$$

while the remaining is lost

$$
\tilde{R} \tilde{\Delta}(a \rtimes L) \tilde{R}^{-1}=\left(\left(R_{1} \triangleright a\right) \rtimes L_{(2)}\right) \otimes_{\mathcal{A}}\left(1_{A} \rtimes R_{2} L_{(1)}\right) \neq \tilde{\Delta}^{o p}(a \rtimes L)=\left(\left(R_{2} \triangleright a\right) \rtimes R_{1} L_{(2)}\right) \otimes_{\mathcal{A}}\left(1_{A} \rtimes L_{(1)}\right),
$$

where in the last equation we have used the property $X \otimes_{\mathcal{A}} s(a) Y=t(a) X \otimes_{\mathcal{A}} Y$. Therefore, any Drinfeld twist $F=F_{1} \otimes F_{2} \in H \otimes H$ in the bialgebra $\mathcal{H}$ can be also shifted to the bialgebroid twist $\tilde{F} \in(\mathcal{A} \rtimes \mathcal{H}) \otimes \mathcal{A}(\mathcal{A} \rtimes \mathcal{H})$ by ${ }^{7}$

$$
F \rightarrow \tilde{F}=\left(1_{A} \rtimes F_{1}\right) \otimes_{\mathcal{A}}\left(1_{A} \rtimes F_{2}\right)
$$

which automatically satisfies bialgebroid cocycle and normalization conditions.

Similarly, the construction of Brzezinski-Militaru (Theorem [I.1 [16]) makes $\mathcal{A}_{F} \rtimes \mathcal{H}^{F}$ a bialgebroid over the algebra $\mathcal{A}_{F}$ if we set

$$
\widetilde{\Delta^{F}}(a \rtimes L)=\left(a \rtimes L_{\left(1^{F}\right)}\right) \otimes_{\mathcal{A}_{F}}\left(1_{A} \rtimes L_{\left(2^{F}\right)}\right), \quad s^{F}(a)=a \rtimes 1_{H}, \quad t^{F}(a)=\left(R_{2}^{F} \triangleright a\right) \rtimes R_{1}^{F}
$$

where $R^{F}=F_{21} R F^{-1}=F_{2^{\prime}} R_{1} \bar{F}_{1^{\prime \prime}} \otimes F_{1^{\prime}} R_{2} \bar{F}_{2^{\prime \prime}}$ and the algebra $\mathcal{A}_{F}$ is braided commutative as well: $a \star_{F} b=$ $\left(R_{2}^{F} \triangleright b\right) \star_{F}\left(R_{1}^{F} \triangleright a\right)$. More explicitly

$$
\widetilde{\Delta^{F}}(a \rtimes L)=\left(a \rtimes F_{1} L_{(1)} \bar{F}_{1^{\prime}}\right) \otimes_{\mathcal{A}_{F}}\left(1_{A} \rtimes F_{2} L_{(2)} \bar{F}_{2^{\prime}}\right)
$$

As a next task, according to $\mathrm{Xu}$ (Theorem โ.2), one applies bialgebroid twisting in order to obtain new twisted bialgebroid $(\mathcal{A} \rtimes \mathcal{H})^{\tilde{F}}$ by making use of the twist $(\underline{25)}$ :

$$
\tilde{\Delta}_{\tilde{F}}(a \rtimes J)=\tilde{F}^{\#}\left(\tilde{\Delta}(a \rtimes J) \tilde{F}^{-1}\right), \quad s_{\tilde{F}}(a)=\left(\bar{F}_{1} \triangleright a\right) \rtimes \bar{F}_{2}, \quad t_{\tilde{F}}(a)=\left(R_{2} \bar{F}_{2^{\prime}} \triangleright a\right) \rtimes R_{1} \bar{F}_{1^{\prime}}
$$

where, in our case, $\tilde{F}^{\#}:(\mathcal{A} \rtimes \mathcal{H}) \otimes_{\mathcal{A}}(\mathcal{A} \rtimes \mathcal{H}) \rightarrow(\mathcal{A} \rtimes \mathcal{H}) \otimes_{\mathcal{A}_{F}}(\mathcal{A} \rtimes \mathcal{H})$ is determined by the formula (cf. (17))

$$
\tilde{F}^{\#}\left((a \rtimes L) \otimes_{\mathcal{A}}(b \rtimes J)\right)=\left(\left(\left(F_{1}\right)_{(1)} \triangleright a\right) \rtimes\left(F_{1}\right)_{(2)} L\right) \otimes_{\mathcal{A}_{F}}\left(\left(\left(F_{2}\right)_{(1)} \triangleright b\right) \rtimes\left(F_{2}\right)_{(2)} J\right)
$$

Since $\left(1_{A} \rtimes M\right) \triangleright a=M \triangleright a$ for any $M \in H$ the base algebra is just $\mathcal{A}_{F}$.

Our goal is to compare bialgebroids $\mathcal{A}_{F} \rtimes \mathcal{H}^{F}$ and $(\mathcal{A} \rtimes \mathcal{H})^{\tilde{F}}$. In fact we are going to prove the following

Theorem III.1 Let $(\mathcal{H}, R)$ be a quasi-triangular bialgebra and $\mathcal{A}$ stands for braided commutative module algebra w.r.t. $(\mathcal{H}, R)$. Assume that $F=F_{1} \otimes F_{2} \in H \otimes H$ is a normalized cocycle twist in $\mathcal{H}$. Then

$$
\mathcal{A}_{F} \rtimes \mathcal{H}^{F} \cong(\mathcal{A} \rtimes \mathcal{H})^{\tilde{F}}
$$

are isomorphic bialgebroids, where $\tilde{F}$ denotes bialgebroid cocycle twist (25) obtained from $F$.

Proof:

All properties of bialgebroid are fulfilled according to Theorems $\amalg$.1 and $\amalg .2$ The base algebra $\mathcal{A}_{F}$ is the same on both sides. Therefore we can use the isomorphism (20) $\varphi: \mathcal{A}_{F} \rtimes \mathcal{H}^{F} \rightarrow \mathcal{A} \rtimes \mathcal{H}$ of total algebras and at the same time, demonstrate that the following diagram commutes (cf. (10))

$$
\begin{array}{ccc}
\mathcal{A}_{F} \rtimes \mathcal{H}^{F} & \mathcal{A} \rtimes \mathcal{H} \\
\widetilde{\Delta^{F}} \downarrow & \downarrow \tilde{\Delta}_{\tilde{F}} \\
\left(\mathcal{A}_{F} \rtimes \mathcal{H}^{F}\right) \otimes_{\mathcal{A}_{F}}\left(\mathcal{A}_{F} \rtimes \mathcal{H}^{F}\right) \stackrel{\varphi \otimes_{\mathcal{A}^{F}}}{\longrightarrow}(\mathcal{A} \rtimes \mathcal{H}) \otimes_{\mathcal{A}_{F}}(\mathcal{A} \rtimes \mathcal{H})
\end{array}
$$

\footnotetext{
7 Our twist is inverse with respect to the one considered by $\mathrm{Xu}$ in [5].
} 
i.e. $\tilde{\Delta}_{\tilde{F}} \circ \varphi=\left(\varphi \otimes_{\mathcal{A}_{F}} \varphi\right) \circ \widetilde{\Delta^{F}}$.

The coproduct $\tilde{\Delta}_{\tilde{F}}$ can be found in more explicit form as

$$
\tilde{\Delta}_{\tilde{F}}(a \rtimes J)=\left(\left(\left(F_{1}\right)_{(1)} \triangleright a\right) \rtimes\left(F_{1}\right)_{(2)} J_{(1)} \bar{F}_{1^{\prime}}\right) \otimes_{\mathcal{A}_{F}}\left(1_{A} \rtimes F_{2} J_{(2)} \bar{F}_{2^{\prime}}\right)
$$

since $\epsilon\left(\left(F_{2}\right)_{(1)}\right)\left(F_{2}\right)_{(2)}=F_{2}$.

In order to simplify the proof we check the diagram (31) on generators. We begin from

$$
\begin{aligned}
\tilde{\Delta}_{\tilde{F}}\left(\varphi\left(1_{A} \rtimes J\right)\right) & =\tilde{\Delta}_{\tilde{F}}\left(1_{A} \rtimes J\right)=\left(\left(\left(F_{1}\right)_{(1)} \triangleright 1_{A}\right) \rtimes\left(F_{1}\right)_{(2)} J_{(1)} \bar{F}_{1^{\prime}}\right) \otimes_{\mathcal{A}_{F}}\left(1_{A} \rtimes F_{2} J_{(2)} \bar{F}_{2^{\prime}}\right) \\
& =\left(1_{A} \rtimes F_{1} J_{(1)} \bar{F}_{1^{\prime}}\right) \otimes_{\mathcal{A}_{F}}\left(1_{A} \rtimes F_{2} J_{(2)} \bar{F}_{2^{\prime}}\right)=\widetilde{\Delta^{F}}\left(1_{A} \rtimes J\right)
\end{aligned}
$$

Next we check

$$
\tilde{\Delta}_{\tilde{F}}\left(\varphi\left(a \rtimes 1_{H}\right)\right)=\tilde{\Delta}_{\tilde{F}}\left(\left(\bar{F}_{1} \triangleright a\right) \rtimes \bar{F}_{2}\right)=\tilde{F}^{\#}\left(\left(\left(\bar{F}_{1} \triangleright a\right) \rtimes\left(\bar{F}_{2}\right)_{(1)} \bar{F}_{1^{\prime}}\right) \otimes_{\mathcal{A}}\left(1_{A} \rtimes\left(\bar{F}_{2}\right)_{(2)} \bar{F}_{2^{\prime}}\right)\right)=
$$

$\tilde{F}^{\#}\left(\left(\left(\left(\bar{F}_{1}\right)_{(1)} \bar{F}_{1^{\prime}} \triangleright a\right) \rtimes\left(\bar{F}_{1}\right)_{(2)} \bar{F}_{2^{\prime}}\right) \otimes_{\mathcal{A}}\left(1_{A} \rtimes \bar{F}_{2}\right)\right)=\left(\left(\left(\left(F_{1^{\prime \prime}}\right)_{(1)} \bar{F}_{1}\right)_{(1)} \bar{F}_{1^{\prime}} \triangleright a\right) \rtimes\left(F_{1^{\prime \prime}}\right)_{(2)}\left(\bar{F}_{1}\right)_{(2)} \bar{F}_{2^{\prime}}\right) \otimes_{\mathcal{A}_{F}}\left(1_{A} \rtimes \bar{F}_{2^{\prime \prime}} F_{2}\right)$

Since $(\Delta \otimes i d)(F)(\Delta \otimes i d)\left(F^{-1}\right)=1_{H} \otimes 1_{H}$ the last expression simplifies to

$$
\left(\left(\bar{F}_{1} \triangleright a\right) \rtimes \bar{F}_{2}\right) \otimes_{\mathcal{A}_{F}}\left(1_{A} \rtimes 1_{H}\right)=\varphi\left(a \rtimes 1_{H}\right) \otimes_{\mathcal{A}_{F}} \varphi\left(1_{A} \rtimes 1_{H}\right)=\left(\varphi \otimes_{\mathcal{A}_{F}} \varphi\right)\left(\widetilde{\Delta^{F}}\left(a \rtimes 1_{H}\right)\right)
$$

In order to complete the proof one has to check that $\varphi\left(s^{F}(a)\right)=s_{\tilde{F}}(a), \varphi\left(t^{F}(a)\right)=t_{\tilde{F}}(a)$ and $\tilde{\epsilon} \circ \varphi=\tilde{\epsilon}$. It is not difficult to get these equalities.

For example, for the target maps we have

$$
\varphi\left(t^{F}(a)\right)=\left(\bar{F}_{1} R_{2}^{F} \triangleright a\right) \rtimes \bar{F}_{2} R_{1}^{F}=\left(\bar{F}_{1}\left(F_{1^{\prime}} R_{2} \bar{F}_{2^{\prime \prime}}\right) \triangleright a\right) \rtimes \bar{F}_{2}\left(F_{2^{\prime}} R_{1} \bar{F}_{1^{\prime \prime}}\right)=\left(R_{2} \bar{F}_{2^{\prime \prime}} \triangleright a\right) \rtimes R_{1} \bar{F}_{1^{\prime \prime}}=t_{\tilde{F}}(a)
$$

using $R^{F}=R_{1}^{F} \otimes R_{2}^{F}=F_{2^{\prime}} R_{1} \bar{F}_{1^{\prime \prime}} \otimes F_{1^{\prime}} R_{2} \bar{F}_{2^{\prime \prime}}$ and $\bar{F}_{1} F_{1^{\prime}} \otimes \bar{F}_{2} F_{2^{\prime}}=F^{-1} F=1_{H} \otimes 1_{H}$.

Therefore the proof is completed.

\section{A. Comments on crossed product and Hopf-Galois extension}

A smash product $\mathcal{A} \rtimes \mathcal{H}$ is a particular kind of a crossed product algebra $\mathcal{A} \rtimes_{\sigma} \mathcal{H}$, where a convolution invertible map $\sigma: \mathcal{H} \otimes \mathcal{H} \rightarrow \mathcal{A}$ has to satisfy (in $\mathcal{A}$ ) the so-called 2-cocycle

$$
\left[L_{(1)} \triangleright \sigma\left(J_{(1)}, K_{(1)}\right)\right] \sigma\left(L_{(2)}, J_{(2)} K_{(2)}\right)=\sigma\left(L_{(1)}, J_{(1)}\right) \sigma\left(L_{(2)} J_{(2)}, K\right), \quad \sigma\left(J, 1_{H}\right)=\sigma\left(1_{H}, J\right)=\epsilon(J) 1_{A}
$$

as well as twisted module

$$
\left[L_{(1)} \triangleright\left(J_{(1)} \triangleright a\right)\right] \sigma\left(L_{(2)}, J_{(2)}\right)=\sigma\left(L_{(1)}, J_{(1)}\right)\left[\left(L_{(2)} J_{(2)}\right) \triangleright a\right]
$$

conditions for any $a \in \mathcal{A}$ and $L, J, K \in \mathcal{H}$. These properties allow to establish on the vector space $\mathcal{A} \otimes \mathcal{H}$ the structure of unital, associative algebra with the multiplication

$$
(a \otimes L)(b \otimes J)=a\left(L_{(1)} \triangleright b\right) \sigma\left(L_{(2)}, J_{(1)}\right) \otimes L_{(3)} J_{(2)}
$$

This algebra is denoted as $\mathcal{A} \rtimes_{\sigma} \mathcal{H}$ [36]. It has a natural left $\mathcal{A}$ module and right $\mathcal{H}$ comodule structures (the so-called normal basis property), which makes it a $H$-comodule algebra (a coring) with the subalgebra $\mathcal{A} \otimes 1_{H}=\left(\mathcal{A} \rtimes_{\sigma} \mathcal{H}\right)^{c o H}$ composed of coinvariants of the coaction. ${ }^{8}$

Due to this fact it provides a canonical example of Hopf-Galois extension 36 which, in turn, is an algebraic counterpart of a quantum principal bundle 36 38. Various twist deformations of such principal bundles have been proposed recently in [38]. Taking the trivial cocycle $\sigma_{0}(L, J)=\epsilon(L) \epsilon(J) 1_{A}$ one reconstructs the smash product. A natural question which appears now is whether the result of the present section can be extended to the case of nontrivial cocycle $\sigma: \mathcal{H} \otimes \mathcal{H} \rightarrow \mathcal{A}$ ?

8 For a coring $M$ over the Hopf algebra $H$ with the right coaction $\rho: M \rightarrow M \otimes H$ one defines a subalgebra of coinvariant elements, $M^{c o H}=\left\{h \in M: \rho(m)=m \otimes 1_{H}\right\}$. We say that the extension $M^{c o H} \subset M$ is $H$-Hopf-Galois if the map $M \otimes \otimes^{c o H} M \rightarrow M \otimes H$, given by $m \otimes n \mapsto\left(m \otimes 1_{H}\right) \rho(n)$, is bijective [36]. 


\section{LIE ALGEBRA CASE}

Nice and simple illustration of the framework presented in this paper is provided by the Lie algebra $\mathfrak{g}$ itself. It is also important from physical point of view as the symmetries in physics are described by Lie algebras. The Lie algebra $\mathfrak{g}$ can be generalized as $U_{\mathfrak{g}}$ - universal enveloping algebra to a Hopf algebra (with primitive Hopf algebra maps) $\mathcal{U}_{\mathfrak{g}}=\left(U_{\mathfrak{g}}, \Delta_{0}, \epsilon, S_{0}\right)$ over the field $\mathbb{K}=\mathbb{C}$ or $\mathbb{R}$ of complex or real numbers. Through the deformation procedure (see Sec. [IB), which requires extension to $\mathbb{K}[[h]]$, it becomes $\mathcal{U}_{\mathfrak{g}, h}=\left(U_{\mathfrak{g}}[[h]], \Delta, \epsilon, S\right)$ deformed quantum symmetry algebra (quantum group) of the corresponding noncommutative quantum spacetime (=Hopf module algebra). In the case of Lie algebras there is well known correspondence between classical and quantum $r$-matrices

$$
R=1+h r+O\left(h^{2}\right)
$$

where $r \in \mathfrak{g} \otimes \mathfrak{g}$ denotes the classical $r$-matrix satisfying, due to (3), classical Yang-Baxter equation (CYBE). Its skew symmetric part $r-r_{21}$ describes Poisson-Lie structure on the corresponding Lie group. In fact, there are two types of quantum deformations of Lie algebras: triangular (nonstandard) and quasi-triangular (standard). The former corresponds to the situation when $r$ is skew-symmetric, i.e. $r \in \mathfrak{g} \wedge \mathfrak{g}$. In this case existing of cocycle twist is ensured by Drinfeld theorem, even if its explicit form not always is known. Knowing the twist $F \in U_{\mathfrak{g}}[[h]] \otimes U_{\mathfrak{g}}[[h]]$ one can proceed with the deformation procedure and construct quantum r-matrix $R=F_{21} F^{-1}$ which is triangular. Twisting techniques are well developed and very useful in mathematical physics (see e.g. [33]). In contrast, quasi-triangular deformations, which apply to semi-simple Lie algebras, are related to the classical $r$-matrices with the skew-symmetric part satisfying modified classical Yang-Baxter equation (MCYBE).

Representations of Lie algebra $\mathfrak{g}$ provide examples of module algebras via deformation of (commutative) algebra of smooth functions $\mathcal{A}=C^{\infty}(V)$ (=algebra of spacetime coordinates) on the corresponding vector space $V$ in the following way. Given representation $\rho$ induces the action on the vector space $V$ :

$$
\rho: \mathfrak{g} \rightarrow \operatorname{End}_{\mathbb{K}} V \quad \Leftrightarrow \quad \triangleright: \mathfrak{g} \otimes V \rightarrow V
$$

$L \triangleright v \equiv \rho(L)(v)$. This action can be uniquely extended to the action of the entire universal enveloping algebra $\triangleright: U_{\mathfrak{g}} \otimes V \rightarrow V$ (and eventually to its topological extension $\left.U_{\mathfrak{g}}[[h]]\right)$.

Further extension relies on the possibility of replacing $V$ by the commutative algebra of smooth functions on $V$ in the case of finite dimesional representation. Assume $\left\{e_{i}\right\}_{1}^{m}\left(m=\operatorname{dim}_{\mathbb{K}} V\right)$ is some basis providing coordinates for the vectors: $v=x^{i} e_{i}$. Let us denote $\rho(L)=\left[L_{i}^{j}\right]$ as the corresponding matrix. Then we are in position to construct the first order differential operators acting on the manifold $V$ or more exactly on the algebra of its smooth functions $C^{\infty}(V)$ :

$$
\hat{\rho}(L)=-L_{\alpha}^{\beta} x^{\alpha} \partial_{\beta}
$$

which is in fact coordinate independent object. Therefore, it defines an extended action $\triangleright: U_{\mathfrak{g}} \otimes C^{\infty}(V) \rightarrow C^{\infty}(V) .{ }^{9}$ Note that the Leibniz rule

$$
\hat{\rho}(L)(a \cdot b)=\hat{\rho}(L) a \cdot b+a \cdot \hat{\rho}(L) b
$$

for all $a, b \in C^{\infty}(V)$ is automatically satisfied. And since $\hat{\rho}(L)$ is a vector field on $V$ one can make use of the primitive Hopf algebra structure $(\Delta(L)=L \otimes 1+1 \otimes L)$ and rewrite (36) as $L \triangleright(a \cdot b)=\left(L_{(1)} \triangleright a\right) \cdot\left(L_{(2)} \triangleright b\right)$ which provides the module algebra condition over $\mathcal{U}_{\mathfrak{g}}$.

Realization (35) allows to merge the initial Lie algebra $\mathfrak{g}$ with a canonical Heisenberg algebra. Resulting algebra can be represented by the following commutation relations

$$
\begin{aligned}
& {\left[L_{a}, L_{b}\right]=\gamma_{a b}^{c} L_{c}, \quad\left[L_{b}, p_{\nu}\right]=\left(L_{b}\right)_{\nu}^{\alpha} p_{\alpha}, \quad\left[p_{\mu}, p_{\nu}\right]=0} \\
& {\left[L_{a}, x^{\mu}\right]=-\left(L_{a}\right)_{\alpha}^{\mu} x^{\alpha}, \quad\left[p_{\nu}, x^{\mu}\right]=1 \delta_{\nu}^{\mu}, \quad\left[x^{\mu}, x^{\nu}\right]=0}
\end{aligned}
$$

The first line (37) represents a Lie subalgebra which can be identified as a inhomogeneous extension $\mathfrak{i g}_{\rho}$ of the initial Lie algebra $\mathfrak{g}$ with respect to the representation $\rho$. Thus the unital associative algebra generated by the relations

\footnotetext{
9 More generally we can assume that $X$ is a (smooth) $G$-manifold, where $\mathfrak{g}$ denotes Lie algebra of $G$. Then we have the action $U_{\mathfrak{g}} \otimes$ $C^{\infty}(X) \rightarrow C^{\infty}(X)$ provided by so-called Killing vector fields.
} 
(37)-(38) can be introduced as a smash product $\operatorname{Pol}\left[x^{1}, \ldots, x^{m}\right] \rtimes U_{\mathfrak{i g}_{\rho}}$, where $\operatorname{Pol}\left[x^{1}, \ldots, x^{m}\right]=U_{A b\left[x^{1}, \ldots, x^{m}\right]}$ is the same as an enveloping algebra of the Abelian Lie algebra $\left[x^{\mu}, x^{\nu}\right]=0$. This former algebra can be interpreted as an extended (quantum) phase space. It contains Heisenberg algebra (quantum phase space) as a subalgebra $\operatorname{gen}\left\{x^{1}, \ldots, x^{m}, p_{1}, \ldots, p_{m}\right\}=\operatorname{Pol}\left[x^{1}, \ldots, x^{m}\right] \rtimes \operatorname{Pol}\left[p_{1}, \ldots, p_{m}\right]$ which can be interpreted as an algebra of differential operators with polynomial coefficients acting on the space $V$. Natural Hopf action of $U_{\mathfrak{i g}}$ on the module algebra $\operatorname{Pol}\left[x^{1}, \ldots, x^{m}\right]$ is given by the commutators (38): $L_{a} \triangleright x^{\mu}=-\left(L_{a}\right)_{\alpha}^{\mu} x^{\alpha}, p_{\nu} \triangleright x^{\mu}=1 \delta_{\nu}^{\mu}$ and provides a representation of the entire smash product algebra $\operatorname{Pol}\left[x^{1}, \ldots, x^{m}\right] \rtimes U_{\mathfrak{i g}_{\rho}}$ on the space $C^{\infty}(V)$. This representation can be extended, in turn, to a Hilbert space representation by introducing a suitable scalar product.

We would like to point out that while extending the Lie algebra (37) by adding the vector space (38) of some representation we exit beyond the category of Lie algebras. However, we remain in a category of associative unital algebras which include Lie algebras as a subcategory. To be more precise, obtained algebra is not an enveloping algebra of some Lie algebra. In other words the unit as a group-like element cannot belong to any Lie algebra. Consequently, instead of bi- (Hopf) algebras one gets bi- (Hopf) algebroids. Replacing the unit by a central (primitive) Lie-algebraic element we change the structure in such a way that Lie algebra and therefore Hopf algebra are possible. But both structures are not equivalent (isomorphic) in the algebraic sense. We claim that our construction is more natural for physics since in the undeformed case it is related to Quantum Mechanics and representations (infinitesimal version) of the so-called Mackey's imprimitivity systems.

Any Drinfel'd twist in the Hopf algebra $U_{\mathfrak{i g}_{\rho}}$ can be used to deforme the smash product algebra $\operatorname{Pol}\left[x^{1}, \ldots, x^{m}\right] \rtimes U_{\mathfrak{i g}_{\rho}}$ in two equivalent ways as described by Theorem III.1 in order to obtain new quantum phase space. In the process of twist deformation (Sec. IIB) (requiring extension of all objects and morphisms to the category of modules over $\mathbb{K}[[h]]$ ring) the Hopf algebra $U_{\mathfrak{i g}_{\rho}}$ gets new coproduct and antipods while the underlying module algebra $P o l\left[x^{1}, \ldots, x^{m}\right]$ gets new twist deformed (noncommutative) star product:

$$
a \star_{F} b=m \circ F^{-1} \triangleright(a \otimes b)=\left(\bar{F}_{1} \triangleright a\right) \cdot\left(\bar{F}_{2} \triangleright b\right)
$$

replacing ordinary (commutative) multiplication of scalar-valued functions. Two-cocycle condition guarantees associativity of the corresponding twisted star-product (39). Note that the twisted star product $\star_{F}$ is braided commutative. One can check that using the relation: $R=F_{21} F^{-1}=F_{2} \bar{F}_{1^{\prime}} \otimes F_{1} \bar{F}_{2^{\prime}}=R_{1} \otimes R_{2}$. Starting from the definition of braided commutativity (4) then from definition of star product (39)

$$
\begin{aligned}
\left(R_{2} \triangleright b\right) \star_{F}\left(R_{1} \triangleright a\right) & =\left(\bar{F}_{1} \triangleright R_{2} \triangleright b\right) \cdot\left(\bar{F}_{2} \triangleright R_{1} \triangleright a\right)= \\
\left(\bar{F}_{1} F_{1^{\prime}} \bar{F}_{2^{\prime \prime}} \triangleright b\right) \cdot\left(\bar{F}_{2} F_{2^{\prime}} \bar{F}_{1^{\prime \prime}} \triangleright a\right) & =\left(\bar{F}_{2^{\prime \prime}} \triangleright b\right) \cdot\left(\bar{F}_{1^{\prime \prime}} \triangleright a\right)= \\
\left(\bar{F}_{1^{\prime \prime}} \triangleright a\right) \cdot\left(\bar{F}_{2^{\prime \prime}} \triangleright b\right) & =a \star_{F} b
\end{aligned}
$$

the second line equality is due to the identity: $1 \otimes 1=F^{-1} F=\bar{F}_{1} F_{1^{\prime}} \otimes \bar{F}_{2} F_{2^{\prime}}$ and the third is using the $\cdot$ commutativity and definition of $\star_{F}$ product.

Therefore, $\mathcal{A}_{F}=\left(A, \star_{F}\right) \in \mathcal{U}_{\mathfrak{g}, h} \mathfrak{Y} \mathfrak{D}^{\mathcal{U}_{\mathfrak{g}, h}}$, hence $\mathcal{A}_{F} \rtimes \mathcal{U}_{\mathfrak{g}, h}^{F}$ can be equipped with the bialgebroid structure (with all the maps as defined in (II.1). Besides the triangular deformations one can consider smash product algebras based on quasi-triangular ones. However, in this case we may not know quantum r-matrix $R$ explicitly (e.g. in the case of non-semi-simple Lie algebras) and then we are unable to check braided commutativity of the corresponding module algebra. This happens, e.g. in the case of the celebrated $\kappa$-Poincare symmetry (see e.g. [39] and references therein). Recently it has been proposed in this case to construct Hopf algebroid which is based on Heisenberg double instead of the smash product construction [2].

Many examples of the smash product algebras, for specific Lie algebras have been already investigated before. For example, the $\kappa$-deformation by twists providing $\kappa$-Minkowski algebra:

$$
\left[\hat{x}^{i}, \hat{x}^{j}\right]=0, \quad\left[\hat{x}^{0}, \hat{x}^{i}\right]=\frac{i}{\kappa} \hat{x}^{i}
$$

as a covariant quantum space of the extended symmetries and their smash products was investigated in [13] for $\mathcal{U}_{\mathfrak{i g l}(n), h^{F}}^{F}$-inhomogeneous general linear algebra, in [40] for $\mathcal{U}_{\mathfrak{i p \mathfrak { w }}, h^{-}}^{F}$ Poincaré-Weyl algebra (one generator extension of Poincaré algebra) or in [41] for the case of $\mathcal{U}_{\mathfrak{s o}(2,4), h}^{F}$ - the conformal algebra.

Such smash product algebras, called extended phase spaces, contain deformed quantum-mechanical phase space (i.e. $\left[P_{\mu}, \hat{x}_{\nu}\right]$ commutators). The deformation of the momenta - coordinates sector leads to the deformation of the Poincaré Casimir operator and therefore to the deformation of dispersion relations. It is due to the fact that the standard Casimir operator $P^{2}$ of the Poincaré algebra does no longer satisfy:

$$
\left[P^{2}, \hat{x}_{\mu}\right] \neq 2 P_{\mu}
$$


once the phase space is deformed. One then looks for another invariant operator - deformed Casimir operator $C_{\kappa}$ for which :

$$
\left[M_{\mu \nu}, C_{\kappa}\right]=\left[C_{\kappa}, P_{\mu}\right]=0 ; \quad\left[C_{\kappa}, \hat{x}_{\mu}\right]=2 P_{\mu}
$$

It will lead to deformed dispersion relation of the form:

$$
C_{\kappa}+m_{\kappa}^{2}=0
$$

(for consequences of this effect see e.g. 21], for discussion of the triangular case, see e.g. 22] and references therein).

In this framework the deformation of the Poincaré Casimir operator (and the corresponding deformation of dispersion relations) is motivated by the use of the noncommutative coordinates assuming that the relations (42) are pre-

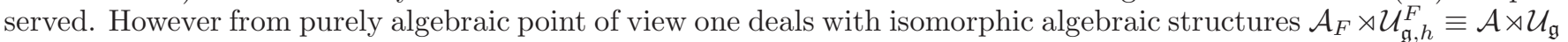
therefore both commuting and noncommuting coordinates are equally justified.

We argue in this paper that distinguishing between noncommutative coordinates can be in turn dictated by the choice of bialgebroid structure. In such approach the deformed extended phase space $\mathcal{A}_{F} \rtimes \mathcal{U}_{\mathfrak{g}, h}^{F}$ is not isomorphic to the undeformed one $\mathcal{A} \rtimes \mathcal{U}_{\mathfrak{g}}$ as bialgebroids, therefore the corresponding deformed coordinates are preferred.

\section{CONCLUSIONS}

The Hopf algebroids (bialgebroids) only recently have gained attention from mathematical physics point of view. In this note we focus on one of the physically important cases where the bialgebroid structure arises, i.e. the smash product construction. We focus on the smash product of triangular Hopf algebra with the Yetter-Drinfeld module algebra and then on the smash product of their twist deformed counterparts. On the other hand we investigate the Drinfeld twist techniques in a category of bialgebroids. We prove that these two approaches, i.e. the twisting of a bialgebroid or constructing bialgebroid from twisted bialgebra are isomorphic in the case of normalized cocycle twist.

As a special example we presented how to obtain Hopf algebroid from Lie algebra. As it is known Lie algebras have special role in physics and many of them have been considered already in the Hopf algebras framework. The smash product algebras of symmetry algebra (i.e. their corresponding Hopf algebra) with the noncommutative coordinates (Hopf) module algebra are called extended phase spaces. In the case of the twist deformation, such extended phase spaces can easily be equipped with the bialgebroid structure as shown in Sec. E. However it is still an open issue if $\kappa$-Minkowski spacetime (40) and $\kappa$-Poincaré (quasi-triangular non triangular case) smash product algebra (true $\kappa$-extended phase space) can be equipped with the Hopf algebroid maps. Some approaches have been made in this direction in [1], [2]. Another two points to consider in the future would be an accommodation of the antipode map within this formalism (knowing it is possible in the case presented in Sec. D and it was already done in [16]) and an extension of the present formalism to the more general cross product construction.

Moreover, there are plenty of other examples of particular interest in physics where the braided commutativity appears naturally, like for example commutative superalgebras (see e.g. [42]). Also the very well known Drinfeld doubles can provide examples of Hopf algebroids (see e.g. [3], Theorem 5.1), i.e. the smash product of the Hopf algebra and the module of its Drinfeld double algebra is Hopf algebroid. More complete presentation of the physically motivated Hopf algebroids as well as more interesting examples we postpone to another paper.

\section{Acknowledgements}

AB was supported by Polish National Science Center (NCN), project 2014/13/B/ST2/04043 and by COST (European Cooperation in Science and Technology) Action MP1405 QSPACE. AP acknowledges the funding from the European Union's Horizon 2020 research and innovation programme under the Marie Sklodowska-Curie grant agreement No 660061. The authors appreciate conversations with Alessandro Ardizzoni, Isar Goyvaerts and Paolo Saracco at the Department of Mathematics 'Giuseppe Peano' University of Turin. We are grateful to Zoran Skoda for numerous discussions and critical remarks.

[1] S. Meljanac, A. Samsarov, R. Strajn, Kappa-deformation of phase space; generalized Poincare algebras and R-matrix, JHEP 1208 (2012) 127 arXiv:1204.4324.

T. Juric, S. Meljanac, R. Strajn, K-Poincare-Hopf algebra and Hopf algebroid structure of phase space from twist, Phys. Lett. A377, 2472 (2013) arXiv:1303.0994. 
T. Juric, S. Meljanac, R. Strajn, Twists, realizations and Hopf algebroid structure of kappa-deformed phase space, Int. J. Mod. Phys. A29, 1450022 (2014) arXiv:1305.3088.

T. Juric, D. Kovacevic, S. Meljanac, kappa-Deformed Phase Space, Hopf Algebroid and Twisting, SIGMA 10, 106 (2014) arXiv:1402.0397.

S. Meljanac, Z. Skoda, M. Stojic, Lie algebra type noncommutative phase spaces are Hopf algebroids, arXiv:1409.8188.

[2] J. Lukierski, Z. Skoda, Mariusz Woronowicz, "kappa-deformed covariant quantum phase spaces as Hopf algebroids", Phys. Lett. B 750 (2015), 401-406 arXiv:1507.02612.

J. Lukierski, Z. Skoda, Mariusz Woronowicz, "On Hopf algebroid structure of kappa-deformed Heisenberg algebra" to appear in Proceedings of IX-thInternational Symposium "Quantum Theory and Symmetries" (QTS-9), held July 13-18, 2015, Yerevan; to be published in "Physics of Atomic Nuclei" (English Version of "Jadernaja Fizika"), ed. G. Pogosyan.

[3] Jiang-Hua Lu, Int. J. Math., 07, 47 (1996) arXiv:q-alg/9505024].

[4] P. Schauenburg, Bialgebras over noncommutative rings and structure theorems for Hopf bimodules, Appl. Categ. Structures 6 (1998), 19317222

[5] Ping Xu, Commun.Math.Phys. 216 (2001) 539-581 arXiv:math/9905192.

[6] M. Sweedler, Groups of simple algebras, I.H.E.S, Publ. 44 (1974), 79-189.

[7] M. Takeuchi, Groups of algebras over $A \bar{\otimes} A$. , J. Math. Soc. Japan 29 (1977), 459-492.

[8] G. Böhm, K. Szlachányi, Hopf algebroid symmetry of abstract Frobenius extensions of depth 2, Commun. Algebra 32 (2004) 4433-4464.

G. Böhm, Hopf algebroids. Handbook of algebra, Vol. 6, 17317235, Handb. Algebr., 6, Elsevier/North-Holland, Amsterdam, 2009.

[9] J. Lukierski, A. Nowicki, H. Ruegg and V. N. Tolstoy, Q-deformation of Poincaré algebra, Phys. Lett. B264 (1991) 331; J. Lukierski, A. Nowicki and H. Ruegg, New quantum Poincaré algebra and $\kappa$-deformed field theory, Phys. Lett. B293 (1992) 344.

[10] S. Majid, H. Ruegg, Bicrossproduct structure of $\kappa$-Poincare group and non-commutative geometryPhys, Lett. B334, 348 (1994) arXiv:hep-th/9405107.

[11] J. Lukierski, A. Nowicki, Heisenberg double description of -Poincare algebra and kappa-deformed phase space,in Quantum Group Symposium of Group 21: Proceedings of the XXI International Colloquium on Group Theoretical Methods in Physics, Editors V.K. Dobrev and H.D. Doebner, Heron Press, Sofia, 1997, 186-192, q-alg/9702003.

Lukierski J., Deformed quantum relativistic phase spaces -an overview, in Proceedings of III International Workshop Classical and Quantum Integrable Systems (Yerevan, 1998), Editors L.D. Mardoyan et al. JINR Dubna Publ. Dept., 1999, 141-152, hep-th/9812063.

[12] P. Kosinski, P. Maslanka "The Kappa-Weyl group and its algebra" Proceedings of the XXII Max Born Symposium

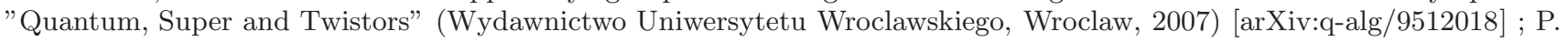
Kosinski, P. Maslanka, Phys.Rev. D68:067702 (2003), arXiv:hep-th/0211057].

[13] A. Borowiec, A. Pachol, $\kappa$-Minkowski Spacetimes and DSR Algebras: Fresh Look and Old Problems, SIGMA 6 , 086 (2010) arXiv:1005.4429.

[14] M. Arzano, F. Nettel, Deformed phase spaces with group valued momenta, arXiv:1602.05788.

[15] S. Zakrzewski, Quantum Poincaré group related to the kappa-Poincar17 algebra, J. Phys. A27, 2075 (1994).

[16] T. Brzezinski, G. Militaru, Bialgebroids, $\times_{A}$-Bialgebras and Duality Journal of Algebra 251, 279-294 (2002)

[17] A. Klimyk, K. Schmüdgen 1997, Quantum groups and their representations (Berlin: Springer-Verlag)

[18] C. Kassel 1995, Quantum groups (New York: Springer-Verlag)

[19] S. Majid, Cross product quantisation, nonabelian cohomology and twisting of Hopf algebras, in Generalized Symmetries in Physics (Clausthal, 1993), World Sci. Publ., River Edge, NJ, 1994, 13-41. [arXive hep-th/9311184].

[20] A. Borowiec, A. Pachol, Bicrossproduct construction versus Weyl-Heisenberg algebra, J. Phys.: Conf. Ser. 343 (2012) 012090

[21] A. Borowiec, Kumar S. Gupta, S. Meljanac, A. Pachol, "Constraints on the quantum gravity scale from kappa - Minkowski spacetime", Europhys.Lett.92:20006 (2010) arXiv:0912.3299

[22] G. Fiore, "On second quantization on noncommutative spaces with twisted symmetries", J. Phys. A: Math. Theor. 43, 155401 (2010) arXiv:0811.0773

[23] H. S. Snyder, Quantized Space-Time, Phys. Rev. 71, 38 (1947);

[24] S. Doplicher, K. Fredenhagen, J.E. Roberts, The quantum structure of spacetime at the Planck scale and quantum fields, Commun. Math. Phys. 172, 187 (1995); arXiv:hep-th/0303037.

[25] C. Bastos, O. Bertolami, N. Costa Dias, J. Nuno Prata, Weyl-Wigner Formulation of Noncommutative Quantum Mechanics, J.Math.Phys.49:072101,2008, arXiv:hep-th/0611257.

[26] Z. Kuznetsova, F. Toppan, Effects of Twisted Noncommutativity in Multi-particle Hamiltonians, Eur.Phys.J. C73 (2013) 2483 e-Print: arXiv:1301.5501 [hep-th].

[27] S. Majid, Quasitriangular Hopf Algebras and Yang-Baxter Equations, Int. J. Mod. Phys. A, 05, 1 (1990).

[28] S. Majid, Foundations of Quantum Group Theory, Cambridge University Press, ISBN 9780521648684, http://dx.doi.org/10.1017/CBO9780511613104.

[29] V. Drinfeld., Quantum groups, in Proceedings of the International Congress of Mathematicians (Berkeley, 1986), Amer. Math. Soc., Providence, RI, 1987, 798-820.

Drinfeld V., Hopf algebras and the quantum Yang-Baxter equations, Sov. Math. Dokl. 32 (1985), 254-258.

[30] D. N. Yetter, Quantum groups and representations of monoidal categories, Math. Proc. Cambridge Philos. Soc. 108 (1990), 261-290. 
[31] L. A. Lambe and D. E. Radford, Algebraic aspects of the quantum Yang-Baxter equation, J. Algebra 154 (1992), $228-288$.

[32] D. E. Radford and J. Towber, Yetter-Drinfeld categories associated to an arbitrary bialgebra. Journal of Pure and Applied Algebra 87 (1993) 259-279.

[33] P. Aschieri, C. Blohmann, M. Dimitrijević, F. Meyer, P. Schupp, J. Wess, A Gravity Theory on Noncommutative Spaces , Class. Quantum Grav. 22, 3511 (2005), arXiv:hep-th/0504183.

P. Aschieri, M. Dimitrijević, F. Meyer, J. Wess, Noncommutative Geometry and Gravity, Class. Quant. Grav. 23, 1883 (2006), arXiv:hep-th/0510059. P. Aschieri, M. Dimitrijević, F. Meyer, S. Schraml, J. Wess, Twisted Gauge Theories, Lett.Math.Phys.78:61-71 (2006) arXiv:hep-th/0603024.

[34] A. Borowiec, A. Pachol, $\kappa$-Deformations and Extended $\kappa$-Minkowski Spacetimes, SIGMA 10 (2014), 107, arXiv:1404.2916.

[35] D. Bulacu, F. Panaite, F. Van Oystaeyen, Generalized Diagonal Crossed Products and Smash Products for Quasi-Hopf Algebras. Applications, Commun. Math. Phys. 266, 35517399 (2006)

F. Panaite, Invariance under twisting for crossed products, Proceedings of the American Mathematical Society, Volume 140, Number 3, 75517763, 2012.

[36] R.J. Blattner, S. Montgomery, Crossed products and Galois extensions of Hopf algebras, Pacific J. Math. 137 (1989), no. 1,371754 .

S. Montgomery, Hopf Galois theory: a survey. New topological contexts for Galois theory and algebraic geometry (BIRS 2008), 36717400, Geom. Topol. Monogr., 16, Geom. Topol. Publ., Coventry, 2009.

[37] T. Brzeziński and S. Majid, Quantum group gauge theory on quantum spaces, Commun. Math. Phys. 157 (1993) 591-638; Erratum-ibid. 167 (1995) 235.

[38] P. Aschieri, P. Bieliavsky, C. Pagani, A. Schenkel, Noncommutative principal bundles through twist deformation, Commun. Math. Phys. arXiv:1604.03542 DOI: 10.1007/s00220-016-2765-x.

[39] A. Borowiec, J. Lukierski, A. Pachol, Twisting and kappa-Poincare, J. Phys. A: Math. Theor. 47405203 (2014) arXiv:1312.7807.

[40] A. Borowiec, A. Pachol, kappa-Minkowski spacetime as the result of Jordanian twist deformation, Phys.Rev.D79:045012 (2009) arXiv:0812.0576

[41] S. Meljanac, A. Pachol, D. Pikutic, "Twisted conformal algebra related to kappa-Minkowski space", Phys. Rev. D 92, 105015 (2015) arXiv:1509.02115.

[42] M. Cohen, S. Westreich, From Supersymmetry to Quantum Commutativity, J. Alg 168, 1-27 (1994). 